\title{
Comparing the Durability and Strength of Simple Adhesive Recipes and PVA-Based Glues
}

\author{
Bethany Elliott ${ }^{1}$ and Sara Fox ${ }^{1}$
}

${ }^{1}$ The Academy of Science and Technology, The Woodlands, TX, USA

\section{ABSTRACT}

Adhesives are essential components of factory production, for maintaining the stability of large structures, and even for pastimes such as paper-mâché crafts. Therefore, it is imperative that adhesives retain their durability and strength when under pressure. The purpose of this project was to test the tensile strength of several adhesive recipes in order to ultimately create a stronger adhesive. To test the tensile strength of each adhesive, one of the adherends had weights attached and was suspended and the amount of time that the adhesive stayed attached was recorded. After completing the experimental procedures, it was found that the flour/water adhesive, cornstarch adhesive, and the waterproof adhesives performed the best, Elmer's glue and the Gum Arabic adhesive performed less well than the first three adhesives, and Elmer's glue with beads did not stay attached. In conclusion, the simple adhesives recipes outperformed Elmer's glue, most likely because they had better cohesive strength and were able to dry faster. The results of this experiment can aid in the creation of a stronger and more cost-efficient adhesive product. Based on these results, future work will be aimed towards determining the effects of environmental changes like humidity and temperature on the tensile strength.

\section{Introduction}

Because adhesives are essential components in so many processes, including factory production, maintaining the stability of large structures, and even simple pastimes such as paper-mâché crafts. Therefore, it is imperative that such adhesives retain their durability and strength when under pressure. For example, skyscrapers that use some form of epoxy adhesive require the adhesive to be able to withstand the pressure created by weight and stresses on the joint over time (Feng, Keong, Hsueh, Wang, \& Sue, 2005). Without proper durability, the adhesive joints in a skyscraper or other structure would quickly fall apart, risking lives and creating a landscape of crumbling infrastructure. While simple household adhesives have a smaller role than holding buildings together, instead being used to mend broken objects and attach craft materials together, they still require some strength in order for people to use them. The purpose of all adhesives is to not only bind objects together, but to bind them well and for a long period of time.

A variety of tests have been conducted to find ways to increase the strength of common adhesives, including an experiment conducted by researchers in Ireland and Pakistan (Khan, May, Porwal, Nawal, \& Coleman, 2013). They added the nanomaterial graphene to a PVA glue and conducted a tensile test. Their results showed that adding between $0.1 \%$ and $0.7 \%$ of graphene to the overall volume of the formula increased the adhesiveness of polyvinyl acetate by twice as much (Khan et al., 2013).

Another experiment measured the amount of adhesive creep, which is how the adhesive stretched, that occurs when industrial epoxy polymer adhesives are subjected to different environmental conditions (Feng et al., 2005). The tested conditions included varying levels of moisture as well as extreme temperatures. The study showed that as time or temperature increased, the creep compliance of the epoxy also increased, demonstrating that the epoxy's molecules become less cohesive as the environmental conditions became more extreme.

Using the theories of adhesion, scientists have designed a variety of methods to test the effectiveness of polymer adhesives (for more information about these theories, see Appendix A). The main form of stress test is the 
tensile strength test (Khan et al., 2013) which exert stress over the entire adhesive joint, causing it to stretch and pull. Two additional common stress tests are the amount shear stress and peel stress (Tukhliyev, Negmatova, Babakhanova, Soliyev, \& Munavvarkhanov, 2020). Shear tests pull the adhesive horizontally rather than vertically, and peel tests cause an uneven distribution of stress on the adhesive, giving researchers information on how the adhesive functions. Since the following project needed to determine the overall strength of the adhesives, it utilized tensile stress tests.

\section{Research Question, Hypothesis, and Engineering Goal}

Though a variety of studies have been conducted on the resistance of industrial adhesives, almost none of them focused on adhesives used in everyday situations. Adhesives like household polyvinyl acetate glue and paper-mâché paste do not have as much durability as other factory-made glues due to their inconsistency. The goal of this project was to compare the endurance and strength of household adhesives through experimentation. The research question was "What type of adhesive will create the most durable bond?" The researcher hypothesized that household glues or adhesives based off of polyvinyl acetate would have created a more durable bond than the other types of simple adhesives due to the fact that the molecules of polymer glues have strong covalent bonds and utilize the diffusion and mechanical interlocking theories of adhesion (Ghanem \& Lang, 2017). After the various household adhesives were tested, the experimenter created an enhanced glue that surpassed the quality of Elmer's Glue based on the findings from the initial tests.

In the experiment, the independent variable was the type of adhesive tested and the dependent variable was the functioning of the adhesive. Some of the controlled variables were the location of the testing site, the size of the adherends, and the weight of the ball bearing. The results from this experiment could be applied to at-home activities by aiding consumers in the decision of what household adhesive to use.

\section{Methods}

\section{Explanation of Procedures}

The six kinds of glue that were tested include the Flour/Water adhesive, Cornstarch adhesive, Waterproof adhesive, Gum Arabic adhesive, Modified Elmer's Glue, and the control group, regular, unmodified Elmer's Glue. The purpose the procedures was to create the six adhesives and then set up the experiment so the researcher could determine the strength of the adhesives. The Flour/Water glue was a simple paste recipe that would be used in a paper- mâché project. The Cornstarch adhesive was like the Flour/Water glue, as it was a somewhat simple paste as well. The Waterproof adhesive was a kind of gel adhesive that solidifies once dried. The Gum Arabic adhesive was a dense superglue, and the two variants of Elmer's Glue were intended for school or experimental purposes.

\section{Materials}

Table 1. List of Materials.

\begin{tabular}{|c|c|c|}
\hline Materials & Total Amount Used & Adhesive Type/Purpose \\
\hline Water & $1392 \mathrm{~mL}$ & $\begin{array}{c}\text { Flour/Water } \\
\text { Cornstarch } \\
\text { Waterproof } \\
\end{array}$ \\
& & Gum Arabic \\
\hline Flour & & Flour/Water \\
\hline Cornstarch & $946 \mathrm{cc}$ & Cornstarch \\
\hline Corn Syrup & $946 \mathrm{cc}$ & Cornstarch \\
\hline
\end{tabular}




\begin{tabular}{|c|c|c|}
\hline Gelatin (unflavored) & $56 \mathrm{~g}$ & Waterproof \\
\hline White Vinegar & $20 \mathrm{~mL}$ & $\begin{array}{c}\text { Cornstarch } \\
\text { Waterproof }\end{array}$ \\
\hline Glycerine & $40 \mathrm{~mL}$ & $\begin{array}{c}\text { Waterproof } \\
\text { Gum Arabic }\end{array}$ \\
\hline Gum Arabic & $60 \mathrm{cc}$ & Gum Arabic \\
\hline Elmer's School Glue Bottles & 2 bottles $(225 \mathrm{~mL}$ each $)$ & $\begin{array}{c}\text { Modified } \\
\text { Control }\end{array}$ \\
\hline Mini Plastic Beads & $30 \mathrm{cc}$ & Modified \\
\hline Wood Blocks & 12 blocks $(5 \mathrm{~cm}$ cubes $)$ & Experimental Setup \\
\hline Short Wood Plank & 1 plank $(122 \mathrm{~cm}(4 \mathrm{ft})$ by $9 \mathrm{~cm}(3.5$ & Experimental Setup \\
& in), $2 \mathrm{~cm}$ thickness $(0.75 \mathrm{in}))$ & Experimental Setup \\
\hline Ball Bearings & 2 bearings $(2.5 \mathrm{~cm}$ diameter $)$ & Experimental Setup \\
\hline Small Mesh Drawstring Bag & 1 bag & Experimental Setup \\
\hline Screw-In Eyes & 12 eyes $(3 \mathrm{~cm}$ long $)$ & Experimental Setup \\
\hline Spoon & 1 spoon & Experimental Setup \\
\hline Medium-Sized Bowls & 5 bowls & Experimental Setup \\
\hline Measuring Cup & 1 cup & Experimental Setup \\
\hline Stainless Steel Pan & 1 pan & Experimental Setup \\
\hline Supports (Chairs) & 2 chairs & Experimental Setup \\
\hline Timer & 1 timer & Experimental Setup \\
\hline Newspaper Sheets & 10 sheets & \\
\hline
\end{tabular}

\section{Procedures}

The researcher made the flour/water glue using $236 \mathrm{cc}$. flour to $236 \mathrm{~mL}$ water. In one of the bowls, the experimenter stirred the water and flour together with a spoon until the mixture was thick and smooth. This was the finished flour/water glue.

The next adhesive recipe was the cornstarch glue. First, the experimenter poured $178 \mathrm{~mL}$ of water into a pan. The pan was set to medium heat. To the pan, the researcher added $60 \mathrm{cc}$. of cornstarch, as well as $30 \mathrm{~mL}$ of light corn syrup. The last ingredient to go in the pan was $5 \mathrm{~mL}$ of white vinegar. After the water, cornstarch, light corn syrup, and white vinegar were in the pan, they were stirred with the spoon until it became thick. In another bowl, $60 \mathrm{cc}$. cornstarch and $60 \mathrm{~mL}$ water were added and blended. Next, the ingredients from the pan were added smoothly to the bowl so that the mixture remained thick and blended. This was the finished cornstarch glue.

The third adhesive was the waterproof glue. In the pan, the experimenter boiled $88 \mathrm{~mL}$ of water. When the water reached a boiling temperature, the researcher turned off the heat and added 14 grams of unflavored gelatin. The mixture was stirred until the gelatin dissolved, then the experimenter added $30 \mathrm{~mL}$ of white vinegar and $5 \mathrm{~mL}$ of glycerine. The glue was stirred well and poured into another bowl. This was the finished waterproof glue.

The last glue recipe was the Gum Arabic superglue. In a bowl, $15 \mathrm{cc}$. of Gum Arabic (powder), $5 \mathrm{~mL}$ of glycerine, and $2.5 \mathrm{~mL}$ of water were added. The mixture was stirred well. This was the finished Gum Arabic adhesive. When all the glue recipes were made, the researcher took one Elmer's glue bottle and unscrewed the cap. $17 \mathrm{cc}$. of mini plastic beads were put into the Elmer's glue, and the glue cap was replaced.

To set up the lab equipment, the experimenter placed the front of the two chair seats/supports $80 \mathrm{~cm}$ apart so that the chair seats faced each other. The wooden board rested on top of the seats, creating an area underneath to conduct the durability test. The newspapers were spread out underneath the board, between the two supports. The 12 eyes were screwed into the centers of the 12 small wood blocks. 
To conduct the experiment, the experimenter turned the long wooden board right-side up and applied the flour/water glue to the small wood block. The glue was placed on the side opposite of the eye. The researcher applied as much glue to the block as the side surface area required. Then, they glued the small wood block to the center of the board and let it dry for 15 minutes. When the 15 minutes had passed, the experimenter flipped the board over carefully and placed it on top of the supports. The glued-on wood block was then hanging upside-down.

The timer was reset to 0 . The ball bearings were placed in the small drawstring bag and hung on the eye. The experimenter started the timer. They timed how many seconds the flour/water glue held the wood together. If the glue stayed attached for more than 1,800 seconds (30 minutes), the researcher recorded that the adhesive stayed on for 30+ minutes. If the flour/water glue did not hold for more than 1,800 seconds and fell onto the newspaper, the experimenter recorded the time in a table. Additionally, the researcher recorded qualitative observations of the adhesive as the trial progressed.

The experiment had 3 trials to solidify the collected data. The researcher repeated the experimental procedures for each of these trials. Each adhesive type was tested the same way as the flour/water glue. These other adhesives were the Cornstarch glue, Waterproof glue, Gum Arabic superglue, Elmer's School glue, and Elmer's School glue with plastic beads. The Elmer's School glue was the control group of this experiment.

The Engineering section of this project was performed with a modified version of the Waterproof adhesive. Using the Waterproof adhesive recipe, the experimenter added $30 \mathrm{cc}$. of cornstarch and $30 \mathrm{cc}$. of corn syrup to add viscosity. The experimenter performed the trials the same as the trials from the main science experiment, but instead let the enhanced adhesive and Elmer's glue dry for 30 minutes (1800 seconds).

During the experiment, there were no accidents or injuries. A parent/guardian supervised the experiment.

\section{Results}

The results of this experiment were collected by creating each of the adhesives and then testing their tensile strength by spreading the adhesive between two adherends, one of which is suspended upside-down with weights to create tension. The adhesives were timed for how long they held that position before falling. Each of the adhesives was tested in this way. Table 1 displays the time in seconds for each of the adhesives, as well as the average time and standard deviation.

Table 2. Attachment Time of Simple Adhesive Trials.

\begin{tabular}{|c|c|c|c|c|c|}
\hline Type of Adhesive & $\begin{array}{c}\text { Trial 1 Time } \\
\text { (seconds) }\end{array}$ & $\begin{array}{c}\text { Trial 2 Time } \\
\text { (seconds) }\end{array}$ & $\begin{array}{c}\text { Trial 3 Time } \\
\text { (seconds) }\end{array}$ & $\begin{array}{c}\text { Average } \\
\text { (seconds) }\end{array}$ & $\begin{array}{c}\text { Standard De- } \\
\text { viation }\end{array}$ \\
\hline Flour/Water & 1800 & 1800 & 1800 & 1800 & 0 \\
\hline Cornstarch & 1800 & 1800 & 1800 & 1800 & 0 \\
\hline Waterproof & 1800 & 1800 & 1800 & 1800 & 78 \\
\hline Gum Arabic & 85 & 107 & 42 & 0 & 0 \\
\hline Elmer's \& Beads & 0 & 0 & 0 & 555 & 512 \\
\hline Elmer's (Control) & 487 & 494 & 51 \\
\hline
\end{tabular}

Figure 1 displays only the average time in seconds, with the standard deviation shown by the error bars. The standard deviations for the Flour/Water glue, Cornstarch glue, Waterproof glue, and Elmer's glue with beads were 0 because 
the measurements were the same. For the Gum Arabic adhesive, the standard deviation was 27 seconds and the standard deviation for Elmer's glue was 31 seconds.

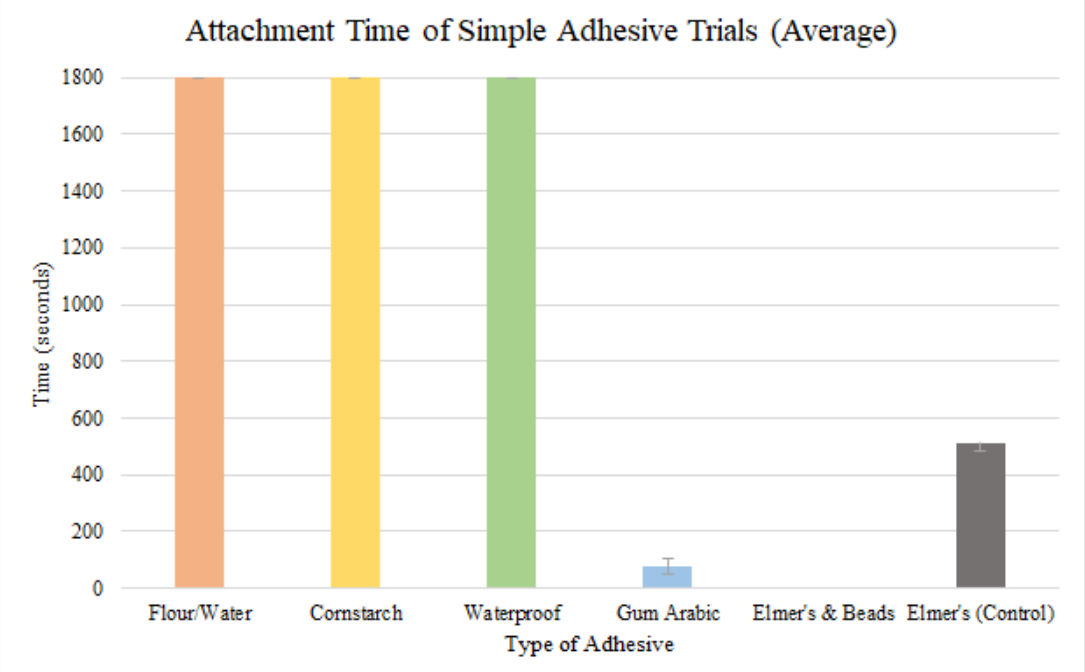

Figure 1. Attachment Time of Simple Adhesive Trials (Average).

For additional statistical analysis, the experimenter performed an ANOVA (Analysis of Variance) test with the adhesives and Elmer's glue.

Null Hypothesis: There is no significant difference between the adhesives and Elmer's glue.

Alternate Hypothesis: There is a significant difference between the adhesives and Elmer's glue.

The data is categorized as significant or not significant with a significance level of 0.05 . The p-value of the ANOVA test was $1.1102 \mathrm{e}-16$, which is a much lower value than 0.05 . The researcher could conclude that there was a significant difference between the adhesives. However, a post-hoc (Tukey HSD) test was needed to determine the differences between groups. The results of the post-hoc test are in Table 3.

Table 3: Results of the Post-Hoc Test.

\begin{tabular}{|c|c|c|c|}
\hline Pair of Samples & $\begin{array}{c}\text { Tukey HSD } \\
\text { Q-Statistic }\end{array}$ & $\begin{array}{c}\text { Tukey HSD } \\
\text { p-value }\end{array}$ & Significant/Insignificant \\
\hline Flour/Water vs Cornstarch & 0 & 0.9 & Insignificant \\
\hline Flour/Water vs Waterproof & 0 & 0.9 & Insignificant \\
\hline Flour/Water vs Gum Arabic & 146.35 & 0.001 & Significant \\
\hline Flour/Water vs Modified Elmer's Glue & 152.98 & 0.001 & Significant \\
\hline Flour/Water vs Elmer's Glue & 109.47 & 0.001 & Significant \\
\hline Cornstarch vs Waterproof & 0 & 0.9 & Insignificant \\
\hline Cornstarch vs Gum Arabic & 146.35 & 0.001 & Significant \\
\hline Cornstarch vs Modified Elmer's Glue & 152.98 & 0.001 & Significant \\
\hline Cornstarch vs Elmer's Glue & 109.47 & 0.001 & Significant \\
\hline Waterproof vs Gum Arabic & 146.35 & 0.001 & Significant \\
\hline Waterproof vs Modified Elmer's Glue & 152.98 & 0.001 & Significant \\
\hline Waterproof vs Elmer's Glue & 109.47 & 0.001 & Significant \\
\hline Gum Arabic vs Modified Elmer's Glue & 6.63 & 0.005 & Significant \\
\hline Gum Arabic vs Elmer's Glue & 36.88 & 0.001 & Significant \\
\hline Modified Elmer's Glue vs Elmer's Glue & 43.51 & 0.001 & Significant \\
\hline
\end{tabular}


For the Engineering section of this experiment, it is shown that the Enhanced adhesive adhered longer than Elmer's glue (see Table 4 and Figure 2). While Table 4 displays all of the data, Figure 2 only shows the average time, and the error bars represent standard deviation.

Table 4: Attachment Time of Enhanced Adhesive and Elmer's Glue.

\begin{tabular}{|c|c|c|c|c|c|}
\hline Type of Adhesive & $\begin{array}{c}\text { Trial 1 Time } \\
\text { (seconds) }\end{array}$ & $\begin{array}{c}\text { Trial 2 Time } \\
\text { (seconds) }\end{array}$ & $\begin{array}{c}\text { Trial 3 Time } \\
\text { (seconds) }\end{array}$ & $\begin{array}{c}\text { Average } \\
\text { (seconds) }\end{array}$ & $\begin{array}{c}\text { Standard Devia- } \\
\text { tion }\end{array}$ \\
\hline Enhanced Adhesive & 1800 & 1800 & 1800 & 1800 & 0 \\
\hline Elmer's (Control) & 590 & 607 & 587 & 595 & 11 \\
\hline
\end{tabular}

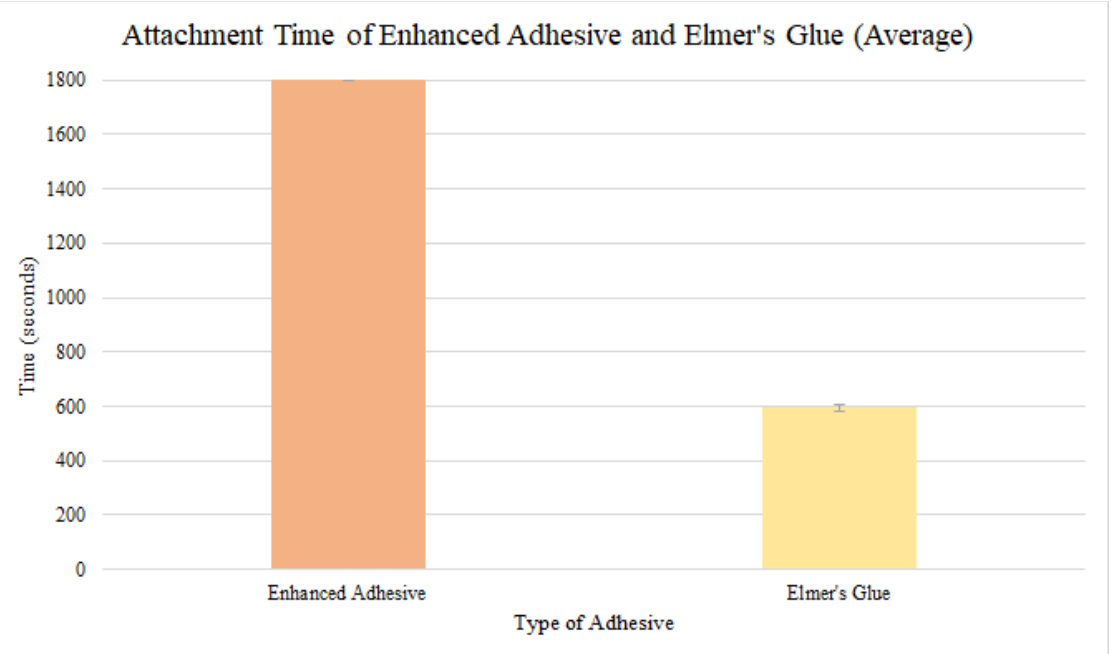

Figure 2: Attachment Time of Enhanced Adhesive and Elmer's Glue (Average)

The experimenter performed a t-test with the enhanced adhesive and Elmer's glue as well. The data is categorized as significant or not significant with a significance level of 0.05 .

Null Hypothesis: There is no significant difference between the enhanced adhesive and Elmer's glue.

Alternate Hypothesis: There is a significant difference between the enhanced adhesive and Elmer's glue.

$$
t=\frac{\left(x_{1}-x_{2}\right)}{\sqrt{\frac{s_{1}{ }^{2}}{n_{1}}}+\frac{s_{2}{ }^{2}}{n_{2}}}
$$

The researcher performed the t-test, and the result was that there was a significant difference between the Enhanced Adhesive and Elmer's Glue. The researcher rejected the null hypothesis.

\section{Discussion}

This experiment was conducted to test how well simple adhesives would adhere compared to a PVA-based adhesive, based off of the hypothesis that PVA-based glues would perform better. The experimental procedures, which involved the measurement of time that the independent variable adhesives could withstand under tensile stress, determined that the Flour/Water adhesive, Cornstarch adhesive, and Waterproof adhesive stayed attached to the adherends for 1800 or greater seconds, longer than any of the other adhesives. Another surprising fact was that neither the Gum Arabic superglue nor the Elmer's glue lasted as long as the homemade recipes. The modified Elmer's glue with beads did not 
stay attached at all. After performing the ANOVA test, the experimenter confirmed that there was a significant difference between the adhesives and rejected the null hypothesis. This means that the performance of the adhesive was directly related to the strength of their formulas, and not due to chance. The post-hoc test determined that some adhesives did display similar qualities, but the researcher believed that this was most likely due to the cut-off time period and not because of similar formulas.

These results did not support the experimenter's hypothesis that household glues or adhesives based off of polyvinyl acetate would create a more durable bond than other types of simple adhesives due to the fact that the molecules of polymer glues have strong covalent bonds and utilize the diffusion and mechanical interlocking theories of adhesion (Ghanem \& Lang, 2017). To support the hypothesis, Elmer's glue had to be the strongest adhesive since it contained polyvinyl acetate. However, this was not the case and half of the simple adhesive recipes appeared to be stronger because their viscosity allowed them to adhere better.

The Engineering section of the experiment determined that the enhanced adhesive made from a modified waterproof adhesive, was stronger than Elmer's glue, which was the control. Thus, the researcher achieved the goal of creating a simple adhesive that is stronger than Elmer's glue. Something to be learned from these trials is that the more viscous the adhesive is, the faster it dried and the better it adhered, as demonstrated by the performance of the viscous enhanced adhesive compared to Elmer's glue (see Figure 2).

One possible reason why Elmer's glue appeared to be weaker was that certain glues require more time to dry in order to reach their full potential. This could also be the same reason why the Gum Arabic adhesive did not perform as well as expected. Long after the trials had concluded, the block which had the Gum Arabic adhesive was not completely dry. The reason why the simple adhesives tended to do better was because they dried faster. This occurrence also appeared in the Engineering section of the project. Predicting this, the experimenter provided 30 minutes of drying time rather than 15 so that Elmer's glue could harden.

Another factor that likely influenced the outcome of this experiment was how much adhesive or cohesive strength each adhesive had. Adhesive strength is how well the adhesive attaches to the adherend, while cohesive strength is how well the molecules of the adhesive stick together (Ghanem \& Lang, 2017). The adhesives that fell off were more adhesive than cohesive. This quality was demonstrated by how the adhesive remains on both of the adherends. For example, the Elmer's Glue with Beads appeared to have a better cohesive strength than adhesive strength because most of the beads in the adhesive still stuck together after the wooden block fell. Perhaps using sand instead of beads could increase the cohesive strength, but that was not tested during the trials.

The results from this project can help consumers who are in need of a simple yet strong solution for multipurpose adhesives. A possible application of the collected data could be to obtain a patent to the engineered adhesive, providing a stronger and possibly more cost-efficient adhesive product. However, the experimental procedures must be corrected to provide more precise results. One extension of this study could be to measure the amount of weight the adhesives could withstand, instead of timing how long the adhesives can withstand tension. Using weight rather than time would improve precision of the measurements. Another extension of this project would be to include the effects of environmental changes to the adhesive. For example, spraying of rinsing the adhesive and its adherends with water could achieve the desired effect of rain. These are just a few possibilities of the future projects.

\section{Conclusion}

In conclusion, simple adhesives have the upper hand in strength and endurance against Elmer's Glue, which represents a polyvinyl acetate adhesive. Thus, the hypothesis was not supported, but the experimenter's engineering goal was achieved. With a quick drying time and excellent cohesiveness, Flour/Water adhesive, Cornstarch adhesive, and Waterproof adhesive stayed attached to their adherends for at least 1800 seconds (30 minutes), while Elmer's glue and the Gum Arabic adhesive only stayed attached for less than 300 seconds ( 5 minutes). Even though there are a few possible errors to the experimental procedures, other variations of these trials could further support the results. There is still much to learn about the binding force of adhesion. 


\section{Limitations}

Despite the positive findings, there were several possible errors within this experiment. One of these errors was the method of timing each adhesive. Because the experimenter's reaction time affected how fast they can stop the stopwatch, the time measurement could be greater by a second or less. This did not affect the result of the experiment as much because the experimenter recorded the time in seconds, not in any fraction of a second. While it would be much more accurate to record the time in tenths of seconds, it would not be reasonable due to human error when stopping the stopwatch. Another error that could have affected the results of the experiment was an error within the creation of the adhesive. Errors in measurement could have affected the trials by making the adhesives inconsistent, influencing the time measurements. This error can be partially corrected by averaging out the measurement times and repeating the trial 3 times. Lastly, the current environment could have affected the trials. Because humidity and temperature affects the performance of some adhesives, it is important to note that the experimental environment is another factor. The environment was mainly controlled due to the indoors location of the experimental setup, but even a house is not resistant to changes in humidity or temperature. This error affects the performance of the adhesives, and like other errors, it can partially be corrected by taking the average of the trials.

\section{Acknowledgements}

I would like to thank Dr. Sara Fox at the Academy of Science and Technology, Mrs. Pawadee Elliott, and Mr. Bryan Elliott for their guidance in the completion of this project.

\section{References}

Feng, C.-W., Keong, C.-W., Hsueh, Y.-P., Wang, Y.-Y., \& Sue, H.-J. (2005). Modeling of long-term creep behavior of structural epoxy adhesives. International Journal of Adhesion and Adhesives, 25(5), 427-436. https://doi.org/10.1016/j.ijadhadh.2004.11.009

Ghanem, A., \& Lang, Y. (2017). Introduction to polymer adhesion. ResearchGate. https://www.researchgate.net/publication/319098070 Introduction to polymer adhesion

Khan, U., May, P., Porwal, H., Nawaz, K., \& Coleman, J. N. (2013). Improved adhesive strength and toughness of polyvinyl acetate glue on addition of small quantities of Graphene. ACS Applied Materials \& Interfaces, 5(4), 1423-1428. https://doi.org/10.1021/am302864f

Pike, R. A. (2015). Adhesive. Encyclopedia Britannica. Retrieved August 30, 2020, from https://www.britannica.com/technology/adhesive

Tukhliyev, G. A., Negmatova, K. S., Babakhanova, M. G., Soliyev, R. Kh., \& Munavvarkhanov, Z. T. (2020). Research of physical-chemical and strength properties of composite Polymer adhesives based on local and secondary raw materials. Journal of Critical Reviews, 7(11), 326-329. http://www.jcreview.com/fulltext/197-1592822661.pdf 